\title{
Relacionamento interpessoal em um centro de atenção psicossocial: articulando educação, design e saúde
}

Interpersonal relationship in a psychosocial care center: articulating education, design and health Relación interpersonal en un centro de atención psicosocial: articulación de educación, diseño y salud

\section{Mislene Beza Gordo Sarzana', Francine Lima Gelbcke ${ }^{\mathrm{II}}$, Melissa Orlandi Honório Locks ${ }^{\mathrm{III}}$, Mônica Stein ${ }^{\text {IV }}$}

Resumo: Objetivo: descrever a prática de intervenção educativa desenvolvida junto a uma equipe multiprofissional em um Centro de Atenção Psicossocial sobre relacionamento interpessoal, inserindo técnicas da área do design thinking para estimular o processo de reflexão. Método: relato de experiência de atividade educativa, em que foram realizados três encontros sequenciais, nos meses de outubro e novembro de 2018, junto a oito profissionais. Resultados: a equipe estava com dificuldade de relacionamento, prejudicando e dificultando o atendimento multiprofissional ao usuário do serviço de atenção psicossocial. As técnicas estimularam a reflexão do grupo a respeito de sua realidade, possibilitando melhorar o relacionamento interpessoal e refletindo positivamente no cuidado prestado ao usuário. Considerações finais: com essa prática foi possível perceber a importância de se articular conhecimentos de outras áreas, como a do design, reforçando os benefícios da busca pela interdisciplinaridade.

Descritores: Relações interpessoais; Saúde mental; Educação em saúde; Enfermagem

Resume: Objective: to describe the practice of educational intervention developed with a multiprofessional team in a Psychosocial Care Center about interpersonal relationships, including design thinking techniques to stimulate the reflection process. Method: experience report of educational activity, in which three sequential meetings were held, in the months of October and November 2018, with eight professionals. Results: the team was having relationship difficulties, impairing and hindering multiprofessional care to users of psychosocial care services. The techniques stimulated the group's reflection about its reality, enabling to improve the interpersonal relationship and reflecting positively on the care provided to the user. Final considerations: with this practice it was possible to realize the importance of articulating knowledge from other areas, such as design, reinforcing the benefits of the search for interdisciplinarity.

\footnotetext{
${ }^{\text {I }}$ Enfermeira. Mestranda em Gestão do Cuidado em Enfermagem pelo Programa de Pós-Graduação em Gestão do Cuidado em Enfermagem Universidade Federal de Santa Catarina - UFSC. Florianópolis, Santa Catarina, Brasil. misbn@hotmail.com. ORCID: https://orcid.org/00000003-2894-9341

II Enfermeira. Doutora em Enfermagem. Universidade Federal de Santa Catarina - UFSC. Florianópolis, Santa Catarina, Brasil. francine.lima@ufsc.com. ORCID: https://orcid.org/0000-0003-3742-5814

III Enfermeira. Doutora em Enfermagem. Universidade Federal de Santa Catarina - UFSC. Florianópolis, Santa Catarina, Brasil. melissa.locks@ufsc.br. ORCID: https://orcid.org/0000-0003-0972-2053

IV Arquiteta e Urbanista. Doutora em Engenharia de Produção. Universidade Federal de Santa Catarina - UFSC. Florianópolis, Santa Catarina, Brasil. moni_stein@yahoo.com.br. ORCID: https://orcid.org/0000-0003-3753-0622
} 
Descriptors: Interpersonal relationships; Mental health; Health education; Nursing

Resumen: Objetivo: describir la práctica de la intervención educativa desarrollada con un equipo multiprofesional en un centro de atención psicosocial sobre las relaciones interpersonales, incluidas las técnicas de pensamiento de diseño para estimular el proceso de reflexión. Método: informe de experiencia de la actividad educativa, en el que se realizaron tres reuniones secuenciales, en los meses de octubre y noviembre de 2018, con ocho profesionales. Resultados: el equipo estaba teniendo dificultades de relación, perjudicando y obstaculizando la atención multiprofesional a los usuarios de los servicios de atención psicosocial. Las técnicas estimularon la reflexión del grupo sobre su realidad, permitiendo mejorar la relación interpersonal y reflexionando positivamente sobre la atención brindada al usuario. Consideraciones finales: con esta práctica fue posible darse cuenta de la importancia de articular el conocimiento de otras áreas, como el diseño, reforzando los beneficios de la búsqueda de la interdisciplinariedad.

Descriptores: Relaciones interpersonales; Salud mental; Educación en salud; Enfermería

\section{Introdução}

O relacionamento interpessoal é uma variável do sistema de administração participativo, que representa o comportamento humano que gera o trabalho em equipe, a confiança e a participação dos envolvidos. ${ }^{1}$ "As pessoas não atuam isoladamente, mas por meio de interações com outras pessoas para poderem alcançar seus objetivos”. 1:115

Os profissionais de saúde utilizam, frequentemente, as relações interpessoais como ferramenta de trabalho para favorecer a comunicação e interação com usuários e entre os colegas da equipe. As necessidades advindas do cotidiano profissional fazem com que estes desenvolvam habilidades sociais como forma de subsidiar os processos de interação. As habilidades sociais podem ser definidas como o conjunto de capacidades comportamentais que são aprendidas e apresentadas pelo indivíduo diante de uma situação interpessoal, incluindo as capacidades de comunicação, resolução de problemas, cooperação, empatia e assertividade. ${ }^{2}$

Dentre os serviços de saúde pensados sob a ótica de uma perspectiva de trabalho multiprofissional e interdisciplinar, encontra-se o Centro de Atenção Psicossocial (CAPS), sendo este um ponto de atenção do componente Atenção Psicossocial Especializada que integra a Rede de Atenção Psicossocial (RAPS). Seu objetivo é realizar atendimentos às pessoas que 
possuem sofrimento psíquico grave e persistente, bem como àquelas que possuam necessidades decorrentes do uso de drogas. ${ }^{3}$

A partir da concepção do CAPS, o atendimento a essa clientela torna-se um desafio não apenas sob o aspecto assistencial como também das próprias relações de trabalho, uma vez que gera desgaste aos trabalhadores, indicando a necessidade de ações específicas voltadas à saúde daqueles que atuam nessa área. ${ }^{4}$ Nesse cenário, é imperativo considerar que os profissionais de saúde são seres interdependentes, que se inter-relacionam e relacionam-se com as pessoas em diversas circunstâncias, bem como dependem de relações sociais, do convívio com amigos, família, colegas de trabalho e com pessoas desconhecidas. ${ }^{5} \mathrm{O}$ relacionamento interpessoal é um fator determinante para o desenvolvimento do cuidado destinado aos pacientes. ${ }^{6}$

A partir de tais conceitos e tomando-se por base o levantamento de um problema de prática que identificou a existência de situações conflitantes entre a equipe de saúde, advindos de uma realidade de um CAPS e, considerando a importância de se criar estratégias que articulem educação, design e saúde como estratégia de minimização de conflitos interpessoais, é que a referida prática educativa foi desenvolvida e aplicada. Para tanto, questiona-se: quais as estratégias da educação e design podem ser utilizadas como ferramenta para melhorar o relacionamento interpessoal de uma equipe multiprofissional de um CAPS I?

Nesse sentido, com esse relato, articula-se a utilização das ferramentas do design thinking como molas propulsoras para reflexão do cotidiano de trabalho no CAPS. Assim, o referido relato tem por objetivo descrever a prática de intervenção educativa desenvolvida junto a uma equipe multiprofissional em um CAPS sobre relacionamento interpessoal, inserindo técnicas da área do design thinking para estimular o processo de reflexão. 
Relacionamento interpessoal em um centro de atenção psicossocial: articulando...| 4

\section{Método}

Trata-se de um relato de experiência sobre prática de intervenção educativa, desenvolvida junto à equipe multiprofissional de um CAPS I, localizado em um município de pequeno porte no Sul do estado de Santa Catarina - Brasil.

A proposta aqui relatada foi desenvolvida a partir da disciplina de Projetos Assistenciais e Inovações Tecnológicas, do Programa de Pós-Graduação Gestão do Cuidado em Enfermagem, modalidade profissional, da Universidade Federal de Santa Catarina, cuja proposta era evidenciar um problema da prática, visando sua resolução. A partir de então, foi identificada a fragilidade de relacionamento interpessoal entre os membros que compõe a equipe de trabalho, que vinha prejudicando e dificultando o atendimento multiprofissional ao usuário do serviço de atenção psicossocial.

A partir desse diagnóstico, foram realizados encontros para reflexão sobre as relações interpessoais, com a participação de oito profissionais da instituição, quais sejam: psicólogo, assistente social, farmacêutico, técnico administrativo, técnico de enfermagem, artesã, auxiliar de serviços gerais, cozinheira.

Primeiramente, os participantes foram convidados de maneira formal, recebendo um convite com a programação de três encontros, os quais ocorreram na sala de reunião do CAPS I. As ações programadas foram desenvolvidas nos meses de outubro e novembro de 2018.

Os encontros pautaram-se na metodologia da problematização - Arco de Maguerez, que consta de cinco etapas - Observação da Realidade; Pontos-chave; Teorização; Hipóteses de Solução e Aplicação à Realidade, ${ }^{7}$ sendo abordados os temas: relação interpessoal, ética, trabalho em equipe e empatia. Para auxiliar as etapas de levantamento dos pontos-chave e de teorização durante a aplicação da metodologia de problematização, foram inseridas técnicas do design thinking para estimular as discussões e buscar soluções, como Mapa Mental e Técnica dos Seis Chapéus, respectivamente. Essas técnicas buscam, por meio de um processo sistemático 
de inovação, priorizar desejos, necessidades e desafios do usuário final para entender um problema, visando desenvolver soluções mais abrangentes e eficazes, levando em consideração o contexto em que esse problema se desenvolve..$^{8-9}$ Especificamente, acerca da técnica do mapa mental, a mesma é bastante difundida no design e é considerado um registro visual ramificado e de informações. Tal estratégia constitui-se "essencialmente por um diagrama hierarquizado de informações, no qual podemos facilmente identificar as relações e os vínculos entre as informações”. ${ }^{10: 4}$

Outra técnica utilizada no segundo encontro foi a técnica dos seis chapéus, que tem como objetivo estimular as pessoas a utilizarem vários raciocínios, de uma maneira sistematizada e disciplinada. Disponibilizam-se chapéus como artefatos físicos que possam ser utilizáveis na prática criativa pelos participantes. Cada um deve sugerir um tipo de raciocínio que o participante deve ter quando os utilizar. O autor dessa técnica associa cada cor com um tipo de raciocínio: o chapéu branco deve procurar ser objetivo e neutro; o chapéu amarelo deve procurar a verdade e a lógica de uma forma otimista; o chapéu verde deve basear-se na criatividade e inovação; o chapéu vermelho deve remeter as emoções, sentimentos e intuições; o chapéu preto deve procurar a verdade por meio da lógica e raciocínio; e o chapéu azul deve assumir a função de controle e avaliação do próprio pensamento. ${ }^{11}$

No quadro a seguir, apresentam-se as etapas do Arco de Maguerez, desenvolvidas nos encontros e as técnicas utilizadas para estimular o processo de reflexão.

Quadro I. Atividades desenvolvidas nos respectivos encontros.

\begin{tabular}{|l|l|l|}
\hline $\begin{array}{l}\text { Ordem } \\
\text { encontros }\end{array}$ & Mês & Atividade desenvolvida \\
\hline $\mathbf{1}^{\mathbf{0}}$ encontro & Outubro & $\begin{array}{l}1^{\underline{a}} \text { Etapa: Observação da realidade } \\
2^{\underline{a}} \text { Etapa: Levantamento dos pontos chaves. Utilização da } \\
\text { técnica do mapa mental. }\end{array}$ \\
\hline $\mathbf{2}^{\mathbf{0}}$ encontro & Novembro & $\begin{array}{l}3^{\underline{a}} \text { Etapa: Teorização } \\
\text { Nessa etapa foi realizada discussão da literatura e } \\
\text { comparando com a realidade da equipe; mensagens e vídeos } \\
\text { motivacionais } \\
4^{\underline{a}} \text { Etapa: Hipóteses de soluções, onde foi utilizada a técnica }\end{array}$ \\
\hline
\end{tabular}


Relacionamento interpessoal em um centro de atenção psicossocial: articulando... 6

\begin{tabular}{|l|l|l|}
\hline & & $\begin{array}{l}\text { dos seis chapéus com o objetivo da equipe sugerir } \\
\text { mudanças para melhorar o relacionamento interpessoal }\end{array}$ \\
\hline $3^{3}$ encontro & Novembro & $\begin{array}{l}5^{\text {a }} \text { Etapa: Aplicação à realidade: houve a discussão das } \\
\text { propostas sugeridas por meio da técnica dos seis chapéus. }\end{array}$ \\
\hline
\end{tabular}

Fonte: Elaborado pelas autoras.

Por se tratar de uma prática assistencial, o projeto não foi submetido ao Comitê de Ética e Pesquisa (CEP), pois tratou-se de atividade educativa e reflexiva do cotidiano de trabalho. Os encontros não foram gravados, portanto, neste texto não se manifestam as falas dos participantes, e sim relata-se o processo de intervenção. No entanto, para garantir a manifestação e ciência dos participantes, foi elaborada uma ata assinada pelos mesmos, afirmando que aceitavam participar da atividade, bem como que receberam informações acerca da proposta. Foi solicitado aos profissionais, por meio de um contrato verbal, o sigilo dos problemas levantados nos encontros, visando garantir a livre manifestação dos participantes.

\section{Problematizando o contexto de trabalho e buscando soluções: Articulando o Arco de Maguerez e o Design Thinking}

Buscar soluções inovadoras num contexto de trabalho nem sempre favorável, exige um método que possibilite a participação ativa dos envolvidos. Neste sentido, a metodologia da problematização foi utilizada sendo que, no primeiro encontro, na etapa "Observação da realidade", os profissionais argumentaram sobre como observavam o relacionamento interpessoal da equipe multiprofissional, identificando o quanto isso estava prejudicado, apontando como fatores a existência de alguns profissionais que apresentavam dificuldade nas relações, manifestado pela falta de empatia, comunicação frágil, falta de respeito, confiança, ética e competitividade.

$\mathrm{Na}$ etapa do método que define os pontos-chave, foram utilizados questionamentos para estimular a reflexão: que tipo de profissional eu sou? Que tipo de profissional eu gostaria de ser? Com que tipo de profissionais eu estou trabalhando? Com que tipo de profissionais eu gostaria 
de trabalhar? Essas questões foram impressas no centro de uma folha A4, permitindo que todos as visualizassem quando apresentadas ao grupo.

Os profissionais receberam post-it coloridos, em que cada cor foi utilizada para responder a um determinado questionamento. Todos os profissionais responderam, conforme a sequência dos questionamentos realizados, destacando-os em forma de palavras-chave importantes que, posteriormente, foram discutidas em equipe e hierarquizadas, gerando um mapa visual, ou seja, um mapa mental.

Em um segundo encontro, ocorreram as etapas do método "Teorização e Hipótese de Solução”. A etapa da teorização foi apresentada em Power point, constituindo-se da apresentação dos aspectos teóricos do CAPS, com um breve histórico sobre sua criação, conceitos de relacionamento interpessoal e maneiras de se trabalhar em equipe. Foram apresentadas também imagens e vídeos com frases de motivação para os participantes.

Na sequência, iniciou-se a etapa de hipóteses de soluções. Para estimular a criatividade dos profissionais, utilizou-se outra técnica utilizada no design, intitulada técnica dos seis chapéus, a qual organiza o processo criativo. No momento da prática, cada profissional recebeu um chapéu e as instruções sobre como utilizá-los para, então, fazer suas observações e contribuições da melhor maneira possível para a equipe trabalhar harmoniosamente.

$\mathrm{Na}$ aplicação da técnica a qual permite ser adaptável a situações diversas conforme o interesse daquele que dela faz uso, o chapéu branco foi utilizado para que fosse identificada a situação atual da equipe Multiprofissional do CAPS-I. Foi constatado que ela encontrava-se fragilizada, sendo apontada a existência de profissionais que assumiam posturas desarmoniosas, a exemplo de manifestações de desrespeito aos profissionais que não possuíam ensino superior, instigando discussões a partir de tais confrontos. 
Relacionamento interpessoal em um centro de atenção psicossocial: articulando... 8

O chapéu verde, por sua vez, permitiu que fosse sugerida a prática do respeito diariamente como um aspecto importante a ser desenvolvido por todos, indiferente da situação. Esse chapéu indicou a comunicação como um aspecto a ser melhorado.

No chapéu vermelho, foram sugeridas dinâmicas para interação entre a equipe, a partir das reuniões quinzenais. Já o chapéu amarelo manteve as sugestões do chapéu vermelho, reforçando as estratégias apontadas.

O chapéu preto foi utilizado de forma que, diante das estratégias sugeridas pelos demais chapéus, fossem indicadas as dificuldades da equipe para promover mudanças, permitindo relações harmoniosas e respeitosas, possibilitando união da equipe em decorrência do comportamento pouco satisfatório de profissionais que apresentam dificuldade para interação, os quais, inclusive, não reconhecem as suas limitações.

O chapéu azul, por fim, foi utilizado para identificar as potencialidades da equipe, bem como as fragilidades que precisam ser resolvidas, começando pelo o respeito entre a equipe multiprofissional. A dinâmica foi encerrada com a concordância de todos acerca das colocações de cada chapéu utilizado.

No último encontro da equipe, o terceiro, foram discutidas as hipóteses de solução, sendo aprofundadas as discussões das propostas levantadas no segundo encontro. A equipe mostrou-se comprometida com a mudança, com os profissionais, almejando essa transformação e reconhecendo que melhores relações implicam de maneira positiva no tratamento ao paciente.

Percebe-se, pós-intervenção prática, um cotidiano profissional já com pequenas alterações nos comportamentos da equipe multiprofissional. Sinais de respeito e reconhecimento pelo outro são visíveis no ambiente de trabalho. Estes confirmam a importância de projetos de intervenção com a utilização de metodologias que promovam a reflexão. 
Destaca-se, ainda, que a aplicação de um projeto de intervenção sobre relação interpessoal na equipe multiprofissional foi bastante desafiador e estimulante, pois foi possível perceber a esperança dos profissionais em melhorar as relações por meio do projeto, motivandoos ao enfrentamento das dificuldades vivenciadas no cotidiano de trabalho. Quanto ao aspecto profissional, é gratificante realizar uma ação e ter um retorno positivo, instigando ainda mais a promoção de mudanças no ambiente de trabalho, ofertando uma dinâmica tranquila, respeitosa, harmoniosa e competente.

\section{Discussão}

O relacionamento interpessoal no local de trabalho é complexo, pois relaciona diversas peculiaridades como autoconhecimento, empatia, autoestima, cordialidade, ética e, principalmente, o diálogo. Considerando essa interação entre pessoas com características diferentes em um ambiente competitivo, faz-se necessário conhecer e compreender o comportamento humano no âmbito das organizações e entender a importância da socialização dentro do trabalho. ${ }^{10}$ Tais relações podem ser afetadas também devido às situações do cotidiano profissional e agravadas pelo ambiente de trabalho em que se encontram; trazendo conflitos com situações estressantes vivenciadas no próprio local de trabalho. ${ }^{6}$

Em um ambiente em que a mudança se faz necessária como nos CAPS que passam pela reorganização de sua prática, as relações interpessoais, por vezes, tornam-se frágeis, interferindo no cotidiano de trabalho e no próprio atendimento ao usuário. ${ }^{4,12}$ Nesse sentido, a prática assistencial desenvolvida foi necessária, pois esteve voltada não só para melhorar a comunicação, mas também tornar a relação interpessoal harmoniosa, implicando na melhoria do atendimento ao usuário, na empatia, paz de espírito, aumento da autoestima e bom-humor.

O cuidado no contexto da saúde mental, engloba as necessidades das pessoas com transtornos mentais e seus familiares. Desse modo, o cuidado de enfermagem tem sua 
significância, pois essa profissão se encontra na maioria dos serviços disponíveis na rede de atenção à saúde. ${ }^{13} \mathrm{~A}$ Enfermagem é uma profissão que está em relação direta com outras profissões, sendo importante a construção e articulação de múltiplos saberes. Salienta-se que na área de saúde mental, com todo o movimento de desinstitucionalização, há uma complexidade a ser reinventada, sendo que o campo da saúde mental é reconhecidamente interdisciplinar, “incorporando um rol enorme de intervenções nas mais diversas áreas”. 14:107 Cogitar sobre as práticas interdisciplinares reflete na valorização dos trabalhadores e, para tanto, há que se criar espaços que possibilitem aos mesmos refletir e dialogar. Acredita-se que a compreensão dos sentimentos vivenciados pelos trabalhadores é necessária para a promoção da saúde dos mesmos, além de refletir diretamente na melhoria da qualidade da assistência prestada. ${ }^{15}$

Para permitir a verbalização e reflexão por parte dos profissionais é necessário estabelecer estratégias que facilitem o processo de comunicação entre os trabalhadores. Nesse sentido, em muitas situações, tem-se que lançar mão de conhecimentos de outras profissões, como foi realizado neste projeto de intervenção. A utilização das dinâmicas do Mapa Mental e da Técnica dos Seis Chapéus, pertinentes à área do design, permitiram a implementação de um espaço criativo em que o trabalhador pode manifestar suas insatisfações, angústias, bem como apontar estratégias que possam contribuir para a mudança no cotidiano de trabalho. A partir do instante que se percebe a importância da interdisciplinaridade, surgem novas perspectivas de cuidado com vistas à construção de um olhar integral, distanciando-se de práticas fragmentadas e descontextualizadas. ${ }^{16}$

Nesse contexto, abordar o tema de relacionamento interpessoal em uma equipe multiprofissional não é uma tarefa fácil, principalmente quando existe no grupo dificuldades relacionais. Dessa forma, há necessidade de um manejo peculiar com o intuito de transformar as atitudes negativas em positivas, promovendo uma melhora no cotidiano profissional, fazendo com que o ambiente de trabalho seja mais harmonioso e prazeroso. Essas ações podem 
11 | Sarzana MBG, Gelbcke FL, Locks MOH, Stein M

beneficiar os cuidados prestados aos usuários, melhorar as relações e permitir que os trabalhadores se sintam atores do processo de trabalho identificando estratégias, bem como colocando-as em prática. Foi identificada fragilidade de relacionamento interpessoal entre os membros que compõe a equipe de trabalho, prejudicando e dificultando o atendimento multiprofissional ao usuário do serviço de atenção psicossocial. Tal posicionamento dos profissionais vinha acarretando intrigas, dificuldade em se relacionarem, ou mesmo postura profissional insatisfatória refletindo, diretamente, nas relações de trabalho e no cuidado prestado.

Há que salientar também a aproximação do design e da saúde cada vez mais presente nos espaços assistenciais, numa perspectiva que articula espaço e usuário, num processo de comunicação em que objetos e ambientes são percebidos como textos e "expressam um modo de perceber o mundo, a relação entre as pessoas e entre estas e os objetos (o produto do design) fazem circular sentidos. Portanto, podem ser considerados mediadores no processo de comunicação entre quem os projeta e quem deles se apropria” 17:2271 São necessários, para tanto, instrumentos que sensibilizem as pessoas para que manifestem a afetividade e possibilitem a reflexão. Experiências similares que desenvolveram oficina piloto, utilizando técnicas como desenho e dramatização, permitindo a escuta sensível dos participantes, puderam concluir e enfatizar sobre a necessidade de novas formas de comunicação. ${ }^{15}$

No relato de experiência aqui apresentado, buscou-se por meio da aproximação com o design utilizar técnicas que permitiram ao trabalhador se expressar, refletir e propor mudanças de forma diferenciada, uma vez que estas se distanciaram das dinâmicas, geralmente, utilizadas na área da saúde. Com a técnica do Mapa Mental, os trabalhadores refletiram sobre seu cotidiano, identificando e registrando de forma visual e hierarquizada os problemas que interferiam nas relações interpessoais. Essa dinâmica foi referendada com a técnica dos Seis 
Chapéus, na qual foram propostas estratégias de enfrentamento para melhorar as relações interpessoais.

Há que se salientar que o design thinking caracteriza-se como uma "abordagem colaborativa de resolução de problemas centrada no usuário, que gera inovação por meio de iteração e práticas criativas”:169 Neste sentido, possibilitou dar voz aos trabalhadores já que essa abordagem está focada no ser humano, nos seus desejos, vontades, necessidades, propiciando novas ideias pautadas no cotidiano, inovadoras e possíveis para se aplicar na prática.

Os resultados das duas dinâmicas tornaram evidente, frente à observação de suas implementações, a importância de se buscar outras disciplinas e técnicas a serem utilizadas nos processos de reflexão por meio do lúdico, dando leveza ao processo e contribuindo para a valorização do trabalhador.

\section{Considerações finais}

A prática de intervenção educativa desenvolvida junto a uma equipe multiprofissional em um Centro de Atenção Psicossocial dentro da perspectiva de relacionamento interpessoal, a partir do uso das técnicas da área do design thinking, mostrou-se factível, inovadora e possibilitou estimular o processo de reflexão. Os profissionais mostraram-se motivados a promover a mudança e esta prática contribuiu sobremaneira para as relações interpessoais saudáveis no ambiente de trabalho.

Logo, reforça-se a importância de se buscar em outras disciplinas, estratégias que estimulem os profissionais a perceberem-se como sujeitos ativos e reflexivos no processo de construção de alternativas para melhoria no processo de trabalho, interferindo no próprio cuidado realizado aos usuários. A partir dessa reflexão aponta-se como fragilidade a lacuna de estudos e outras experiências que abordam a saúde do trabalhador no contexto da saúde mental e as relações interpessoais nessa área de atuação, dificultando comparações e discussões dentro 
dessa temática aqui explorada, corroborando ainda mais a necessidade de novos estudos nesta área do conhecimento.

As técnicas utilizadas contribuem sobremaneira para a formação profissional, uma vez que por meio de um processo sistemático de inovação, prioriza desejos, necessidades e desafios, visando desenvolver soluções mais abrangentes e eficazes. Assim, espera-se que esse relato possa contribuir com outros profissionais não só para a melhoria das relações interpessoais em seu ambiente de trabalho, mas também para que estes possam apreender outras técnicas como as usadas pelo design, a partir das experiências aqui retratadas.

\section{Referências}

1. Chiavenato I. Iniciação à teoria das organizações. São Paulo (SP): Manole; 2010.

2. Ferreira FR, Carvalho MAG, Senem CJ. Desenvolvendo habilidades sociais na escola: um relato de experiência. Constr Psicopedag [Internet]. 2016 [acesso 2019 out 25];24(25):84-98. Disponível em: http://pepsic.bvsalud.org/scielo.php?script=sci_arttext\&pid=S1415-69542016000100007\&lng=pt\&nrm=iso

3. Brasil. Ministério da Saúde. Portaria no 3.088, de 23 de dezembro de 2011. Institui a Rede de Atenção Psicossocial para pessoas com sofrimento ou transtorno mental e com necessidades decorrentes do uso de crack, álcool e outras drogas, no âmbito do Sistema Único de Saúde (SUS) [Internet]. 2013 [acesso em 2018 nov 01]. Disponível em: http://www.saude.pr.gov.br/arquivos/File/Portaria_n_3088_2011_republicada_Rede_RAPS.pdf

4. Bellenzani R, Paro DM, Oliveira MC. Trabalho em saúde mental e estresse na equipe: questões para a política nacional de humanização/SUS. Rev Psicol Saúde [Internet]. 2016 [acesso em 2018 out 15];8(1):3243. Disponível em: http://pepsic.bvsalud.org/scielo.php?script=sci_arttext\&pid=S2177093X2016000100005\&lng=pt\&nrm=iso

5. Baggio MA, Erdmann AL. Processando o cuidado "do nós” nas relações/interações estabelecidas por profissionais de enfermagem e de saúde. Cogitare Enferm [Internet]. 2015 [acesso em 2018 out 10];20(3). Disponível em: https://revistas.ufpr.br/cogitare/article/view/41177

6. Martins CCF, Santos VEP, Pereira MS, Santos NP. Relacionamento interpessoal da equipe de enfermagem x estresse: limitações para a prática. Cogitare Enferm [Internet]. 2014 [acesso em 2018 out 25];19(2). Disponível em: http://dx.doi.org/10.5380/ce.v19i2.36985 
7. França FCV, Melo MC, Monteiro SNC, Guilhem D. O processo de ensino e aprendizagem de profissionais de saúde: a metodologia da problematização por meio do arco de Maguerez. Brasília (DF): Teixeira; 2016.

8. Roberts JP, Fisher TR, Trowbridge MJ, Bent C. A design thinking framework for healthcare manegemant and innovation. Healthc (Amst) [Internet]. 2016 [acesso em 2019 out 16];4(1):11-4. Disponível em: https://www.sciencedirect.com/science/article/pii/S221307641500113X

9. Macedo MA, Miguel PAC, Casarotto Filho N. A caracterização do design thinking como um modelo de inovação. RAI Rev Adm Inov [Internet]. 2015 [acesso em 2019 out 16];12(3):157-82. Disponível em: https://www.sciencedirect.com/science/article/pii/S1809203916300961

10. Ourives EAA, Ferreira AS, Figueiredo LFG, Vieira MLH, Figueiredo LG. O ensino da representação gráfica nos cursos de design: mapas mentais e conceituais, ferramentas de estruturação de conteúdo [Internet]. 12 Congresso Brasileiro de Pesquisa e Desenvolvimento em Design. São Paulo, BR. Anais. (Blucher Proceedings. 2016;2(9):2702-14). 2016 nov [acesso em 2019 out 25]. Disponível em: 10.5151/despro-ped2016-0231

11. De Bono, E. Six thinking hats. Boston: Little, Brown; 1985.

12. Cardozo CG, Silva LOS. A importância do relacionamento interpessoal no ambiente de trabalho. Interbio [Internet]. 2014 [acesso em 2018 out 20];8(2):24-34. Disponível em: https://www.unigran.br/dourados/interbio/paginas/ed_anteriores/index.php?id=artigo\&codigo=\%20108\&c am=vol8_num2

13. Siqueira DF, Xavier MS, Serbim AK, Terra MG. Redes sociais de apoio no cuidado à pessoa com transtorno mental: reflexões. Rev Enferm UFSM [Internet]. 2018 [acesso em 2019 fev 01]. Disponível: http://dx.doi.org/10.5902/2179769226118

14. Vasconcelos EM. Complexidade e pesquisa interdisciplinar: epistemologia e metodologia operativa. 6ª ed. Petrópolis (RJ): Vozes; 2013.

15. Miorin JD, Camponogara S, Pinno C, Beck CLC, Costa V, Freitas EO. Prazer e sofrimento de trabalhadores de enfermagem de um pronto-socorro. Texto \& Contexto Enferm [Internet]. 2018 [acesso em 2019 out 25];27(2):e2350015. Disponível em: http://www.scielo.br/scielo.php?script=sci_arttext\&pid=S0104-07072018000200305\&lng=pt

16. Anjos Filho NC, Souza AMP. The workers' perceptions about the multiprofessional team work at a Psychosocial Care Center in Salvador, Bahia, Brazil. Interface (Botucatu). 2017;21(60):63-76.

17. Grossman E, Araújo-Jorge TC, Araujo IS. Reflexões sobre os objetos e os ambientes físicos de ensino e pesquisa em saúde. Ciênc Saúde Colet [Internet]. 2008 [acesso em 2018 out 25];13(2). Disponível em: http://dx.doi.org/10.1590/S1413-81232008000900031 
15 | Sarzana MBG, Gelbcke FL, Locks MOH, Stein M

\section{Autor correspondente}

Mislene Beza Gordo Sarzana

E-mail: misbn@hotmail.com

Endereço: Rua Manoel Antônio Machado, 216. Braço do Norte, Santa Catarina.

CEP: 88750-000

\section{Contribuições de Autoria}

1 - Mislene Beza Gordo Sarzana

Concepção e planejamento do projeto de pesquisa, análise e interpretação dos dados, redação.

2 - Francine Lima Gelbcke

Análise e interpretação dos dados, redação e revisão crítica.

3 - Melissa Orlandi Honório Locks

Análise e interpretação dos dados, redação e revisão crítica.

4 - Monica Stein

Interpretação dos dados, e revisão crítica.

\section{Como citar este artigo}

Sarzana MBG, Gelbcke FL, Locks MOH, Stein M. Relacionamento interpessoal em um centro de atenção psicossocial: articulando educação, design e saúde. Rev. Enferm. UFSM. 2019 [Acesso em: Anos Mês Dia]; vol.9 e68: 1-15. DOI:https://doi.org/10.5902/2179769237124 